\title{
Structure and Magnetism of Fe/Si Multilayers Grown by Ion-Beam Sputtering
}

\author{
A. Chaikent, R.P. Michelt and M.A. Walle \\ Materials Science and Technology Division \\ Lawrence Livermore National Lab \\ Livermore, CA 94551
}

(October 11, 2018)

\begin{abstract}
Ion-beam sputtering has been used to prepare Fe/Si multilayers on a variety of substrates and over a wide range of temperatures. Small-angle x-ray diffraction and transmission electron microscopy experiments show that the layers are heavily intermixed although a composition gradient is maintained. When the spacer layer is an amorphous iron silicide, the magnetic properties of the multilayers are similar to those of bulk Fe. When the spacer layer is a crystalline silicide with the $\mathrm{B} 2$ or $\mathrm{DO}_{3}$ structure, the multilayers show antiferromagnetic interlayer coupling like that observed in ferromagnet/paramagnet multilayers such as $\mathrm{Fe} / \mathrm{Cr}$ and $\mathrm{Co} / \mathrm{Cu}$. Depending on the substrate type and the growth temperature, the multilayers grow in either the (011) or (001) texture. The occurrence of the antiferromagnetic interlayer coupling is dependent on the crystallinity of the iron and iron silicide layers, but does not seem to be strongly affected by the perfection of the layering or the orientation of the film. Since the B2- and $\mathrm{DO}_{3}$-structure $\mathrm{Fe}_{\mathrm{x}} \mathrm{Si}_{1-\mathrm{x}}$ compounds are known to be metallic, antiferromagnetic interlayer coupling in $\mathrm{Fe} / \mathrm{Si}$ multilayers probably originates from the same quantum-well and Fermi surface effects as in $\mathrm{Fe} / \mathrm{Cr}$
\end{abstract}


and $\mathrm{Co} / \mathrm{Cu}$ multilayers.

75.50.Bb,61.10.-i,68.65.+g,81.15.Cd,75.30.E

Typeset using REVTEX 


\section{INTRODUCTION}

Multilayer films formed from transition metals and semiconductors have long been studied because of their unusual superconducting propertiest and because of possible application as x-ray optical elements. 2 Many unusual phenomena have been produced, ranging from the observation of dimensional crossover in weakly coupled superconducting $\mathrm{Nb}$ layers in $\mathrm{Nb} / \mathrm{Ge}$

multilayersit to the occurrence of bcc Ge in short-period Mo/Ge multilayers. B $^{\text {U }}$ Unusual magnetic properties have recently been observed in Fe/Si multilayers by workers at ETH目 and Argonne.5 A large antiferromagnetic (AF) interlayer coupling in these multilayers manifests itself in hysteresis loops as a high saturation field and a low remanent magnetization. Similar magnetization curves are associated with large interlayer coupling in metal/metal multilayers like $\mathrm{Fe} / \mathrm{Cr}$ and $\mathrm{Co} / \mathrm{Cu} 6$ G $\mathrm{Much}$ consideration has been given to whether the coupling in the $\mathrm{Fe} / \mathrm{Si}$ system has the same origin as in the metal/metal multilayers 896 Therefore the question of whether the spacer layer in the Fe/Si multilayers is a metal or semiconductor is of particular interest.

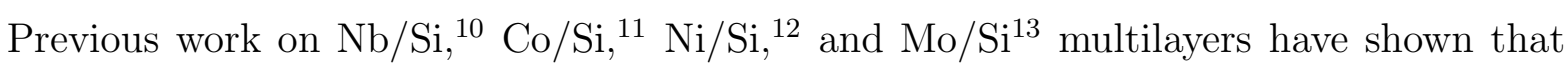
there is a strong tendency towards compound formation at the metal/silicon interface. In general these multilayers consist of polycrystalline metal layers separated by an amorphous silicon layer which is bounded on either side by a layer of intermixed material. The intermixed silicide layers in these films were amorphous unless they were annealed at several hundred ${ }^{\circ} \mathrm{C} .11,14$ These previously studied multilayers were therefore likely in their as-grown state to have metal/semiconductor character because of the presence of the amorphous silicon layer.

In order to investigate the character of the spacer layer in the Fe/Si multilayer system, we have grown a large number of films with different substrate temperatures, substrate types, and layer thicknesses. When the Si spacer layer thickness is greater than about $20 \AA$, we find that the metal layers are crystalline but that the spacer layers are amorphous, similar to the situation in other transition metal/silicon systems. When the Si spacer layer thickness is 
less than about $20 \AA$ Ahick, the iron silicide spacer layer forms a crystalline silicide with either the $\mathrm{B} 2$ or $\mathrm{DO}_{3}$ structure. The $\mathrm{B} 2$ structure consists of two interpenetrating simple cubic sublattices and is identical to the $\mathrm{CsCl}$ structure for a 1:1 ratio of $\mathrm{Fe}$ and $\mathrm{Si}$, 国 while the $\mathrm{DO}_{3}$ structure is an fcc lattice with two inequivalent Fe sites.16 Extensive growth experiments, described below, suggest that crystallinity of the spacer layer is crucial for occurrence of the antiferromagnetic interlayer coupling, in keeping with previous suggestions. $\mathrm{B} 2$ and $\mathrm{DO}_{3}$ phases are metallic, 15,16 the fact that crystallinity is required for antiferromagnetic coupling suggests that the coupling in $\mathrm{Fe} / \mathrm{Si}$ has a common origin with that observed in metal/metal multilayers.

\section{EXPERIMENTAL METHODS}

The Fe/Si multilayers are grown in the ion-beam sputtering (IBS) chamber whose layout is shown schematically in Figure 1. The system base pressure is typically about $2 \times 10^{-8}$ torr. The ion gun is a $3 \mathrm{~cm}$ Kauffman source with focusing optics. 1 . The energy of the ions leaving the gun can be modulated by raising and lowering the voltage on the acceleration grids, creating in effect an electrical shutter. The Ar ions are incident on the sputter target at $1000 \mathrm{~V}$ at an angle of about $45^{\circ}$. The Ar pressure is maintained in the $2-3 \times 10^{-4}$ range by a flow-controller coupled to a capacitance manometer. 18 Four 3" diameter sputter targets are

mounted on a tray which can be rotated by a stepper motor 19 Layer thickness is monitored by a quartz-crystal oscillator which is placed in close proximity to the substrates. The substrates are about $25 \mathrm{~cm}$ above the targets, clamped to a copper tray. The temperature of the tray is monitored by a thermocouple and can be varied between $-150^{\circ} \mathrm{C}$ and $+200^{\circ} \mathrm{C}$. 20 Three films are grown per chamber pumpdown.

The thickness monitor, the controller for the stepper motor and the ion-beam power supply are all interfaced to a personal computer which has been programmed using the ASYST instrument control package.21 When the system is depositing a multilayer, the computer sends the material parameters to the thickness monitor, rotates the stepper motor to 
its new orientation, and turns the ion gun on. When the desired thickness is reached, the thickness monitor turns the ion-gun off and prompts the computer for the next layer. The basic design of the system is similar to one previously described by Kingon et al.22

The substrates for multilayers growth include glass coverslips, oxidized silicon wafers, $\mathrm{MgO}(001)$ and $\mathrm{Al}_{2} \mathrm{O}_{3}(0 \overline{2} 11)$. The first two substrates, which are used for growth of polycrystalline films, were rinsed in solvents before loading into the vacuum chamber. The second two, which are used for epitaxial growth, are cleaned according to a recipe reported by Farrow and coworkers.23 The typical deposition rate for Fe is $0.2 \AA / \mathrm{s}$ while that for Si is about $0.3 \AA /$ s. All films are capped with a $200 \AA$ Ge oxidation barrier. The magnetic and structural properties of the films are stable for at least one year. Ge is used for capping instead of $\mathrm{Si}$ in order to prevent interference with element-specific soft x-ray fluorescence measurements, which will be reported elsewhere.24

X-ray diffraction characterization has been performed using a $18 \mathrm{~kW}$ rotating anode system outfitted with a graphite monochromator. All spectra are taken using the $\mathrm{Cu} \mathrm{K}_{\alpha}$ wavelength. Conventional high-resolution electron microscopy and electron diffraction have been performed in order to characterize the microstructure of the as-deposited films in crosssection. Magnetization curves are obtained using a vibrating sample magnetometer. All the data shown here were taken at room temperature.

\section{RESULTS}

Overall the magnetic properties of the Fe/Si multilayers made by IBS are similar to those made previously by magnetron sputtering. 225 Definitive confirmation of AF interlayer coupling in our multilayers has been obtained by polarized neutron reflectivity measurements.26 For some unknown reason the magnetic properties of our multilayers are closer to those of the magnetron-sputtered multilayers than those reported on in a previous study using IBS, where much lower saturation fields were observed 27 The differences between the previous IBS-grown films and ours may be related to the lower ion-beam voltage used by Inomata et 
al.27 Comparisons on the basis of layer thickness are made here only between films grown during the same deposition run in order to insure that the relative layer thicknesses are meaningful. Films with similar layer thicknesses have been grown many times to establish reproducibility of the observed trends.

\section{A. Layer-Thickness Dependence of Properties}

Forty- and fifty-repeat multilayers have been grown with $\mathrm{t}_{\mathrm{Fe}}=14,20,30,40$, and $50 \AA$ and $\mathrm{t}_{\mathrm{Si}}=14$ and $20 \AA$. Magnetization curves for 50-repeat $(\mathrm{Fe} 30 \AA / \mathrm{Si} 20 \AA)$ and $(\mathrm{Fe} 30 \AA / \mathrm{Si} 14 \AA)$ multilayers grown on glass at nominal RT $\left(\right.$ about $\left.+60^{\circ} \mathrm{C}\right)$ are shown in Figure 2. On the y-axis of this plot is the magnetic moment of the multilayer normalized to the moment of an equivalent volume of bulk Fe. The magnetization curve of the (30/20) multilayer looks much like that of an Fe film, while the magnetization curve of the (30/14) multilayer shows the high saturation field and low remanence which characterize AF interlayer coupling. At its saturation field the magnetization of the (30/14) multilayer is about the same as for the $(30 / 20)$ multilayer. Both of these films have moments only about half as large as an equivalent volume of bulk Fe. Our observation of AF coupling for Si thicknesses between 10 and $20 \AA$ and the disappearance of coupling for Si thicker than $20 \AA$ confirm previous observations on magnetron-sputtered films.

X-ray diffraction spectra for these multilayers are shown in Figures 3 and 5 . Figure 3 shows the small-angle x-ray scattering (SAXS) data with peaks at angles

$$
n^{2} \lambda^{2}=4 \Lambda^{2} \sin ^{2} \theta+2 \delta
$$

where $\lambda$ is the x-ray wavelength, $\Lambda \equiv \mathrm{t}_{\mathrm{Fe}}+\mathrm{t}_{\mathrm{Si}}$ is the multilayer bilayer period and $\delta$

is the index of refraction for x-rays.28 This grazing incidence data gives information about the quality of the multilayer interfaces. Figure 3 shows four low-angle peaks for both films, indicating a reasonably strong composition modulation along the growth direction. (The higher frequency oscillations between $1^{\circ}$ and $4^{\circ}$ are finite-thickness fringes from the Ge cap 
layer.) The most notable difference between the two spectra is that the multilayer peaks are broader for the AF-coupled $\mathrm{t}_{\mathrm{Si}}=14 \AA$ film, indicating more fluctuations in bilayer period and probably more interface roughness. Using the spacing between peak positions to eliminate the unknown $\delta$ from Eqn. 1 gives values of the bilayer period $\Lambda$ for the two films. For the multilayer with nominal layering of $(\mathrm{Fe} 30 \AA / \mathrm{Si} 20 \AA) \times 50$, the derived value for $\Lambda$ is $(41.82 \pm$ 0.07) $\AA$, while for the $(\mathrm{Fe} 30 \AA / \operatorname{Si} 14 \AA) \times 50$ film $\Lambda=(38.10 \pm 0.04) \AA . \Lambda$ is $8.2 \AA$ shorter than the nominal value for the $\mathrm{t}_{\mathrm{Si}}=20 \AA$ film, and $5.9 \AA$ shorter than nominal for the $\mathrm{t}_{\mathrm{Si}}=14 \AA$ film. Although some of the discrepancy between the nominal and observed bilayer period may be due to calibration inaccuracies, most is undoubtedly due to intermixing of the Fe and Si layers, in keeping with observations in the other metal/Si multilayers.10.14 Throughout this paper we will continue for convenience to refer to the films in terms of their nominal layer thicknesses.

Comparison of the magnetization data to the x-ray data can give some further insight into the question of intermixing. Because of the presumed interdiffusion of the Fe and Si layers, the magnetic moment of the Fe layers is also reduced from the nominal value. The missing magnetic moment can be expressed as an equivalent thickness of Fe. Figure 1 shows a plot of missing moment in units of Ångstroms of Fe versus missing bilayer period determined from multilayer peak positions in SAXS for films grown at room temperature (RT). The plot shows that while the diffusion-induced reduction in bilayer period varies between 1 and $8 \AA$, the missing Fe moment per bilayer (for both interfaces) is consistently between 10 and $12 \AA$. The one outlier in Figure G is for a film which had $t_{\mathrm{Fe}}=20 \AA$, the thinnest Fe for which we have ever observed interlayer coupling. Other groups have previously observed a moment reduction of $12-14 \AA$ per bilayer in polarized neutron reflectivity measurements on uncoupled $\mathrm{Fe} / \mathrm{Si}$ multilayers with thick Si layers.29.30

The disparity between the magnetic moment reduction and the bilayer period reduction numbers may at first appear to be puzzling. This disparity occurs because the moment and bilayer period are affected by different aspects of the structure. In calculating the moment reduction in $\AA$ the assumption has been made that the Fe layer has the magnetization of bulk 
Fe. This is equivalent to assuming that there is no $\mathrm{Si}$ in the Fe layer, which is undoubtedly false. In calculating the missing bilayer period, the assumption has also been made that the spacer layer is pure $\mathrm{Si}$, also clearly false. The fact that the missing magnetic moment is almost constant irrespective of the reduction in bilayer period suggests that the spacer layer is non-magnetic independent of Si thickness. The lack of variation of the missing moment is then explained by the diffusion of a constant number of iron atoms into the silicon layer, irrespective of its thickness. The wide variation of the measured bilayer period is most likely related to the varying orientation and crystallinity of the spacer layer, neither of which affects the magnetic moment if the spacer itself is non-magnetic.

Figure 5 shows the high-angle x-ray spectra where peak positions give information about the orientation and crystallinity of the films. The intense peak near $70^{\circ}$ in this plot is due to the Si substrate. Included are data for an $(\mathrm{Fe} 40 \AA / \mathrm{Si} 14 \AA) \times 40$ antiferromagnetically coupled multilayer and for an $(\mathrm{Fe} 30 \AA / \mathrm{Si} 20 \AA) \times 40$ uncoupled multilayer, both grown on oxidized $\mathrm{Si}(001)$ at RT. The peaks for the (40/14) film are narrower than for the $(30 / 20)$. The Scherrer formula gives $78 \AA$ or about two bilayer periods for the coherence length of the (40/14) film and $34 \AA$ or about one bilayer period for the coherence length of the $(30 / 20)$ film. Coherence lengths in IBS-sputtered antiferromagnetically coupled films are often as long as $200 \AA$. Fullerton et al. have inferred that the spacer layer in thin-Si Fe/Si multilayers must be crystalline based on their observation of coherence lengths longer than a bilayer

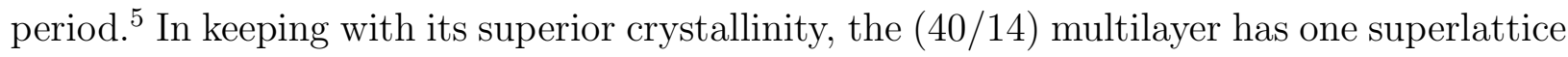
satellite on the low-angle side of the $\mathrm{Fe}(002)$ peak. Typically only one satellite on the lowangle side of the $\mathrm{Fe}(011)$ or (002) x-ray peak is observed for polycrystalline multilayers grown on glass, in agreement with observations by Foiles et al. 19

The thin-Si multilayers which have AF coupling usually show a mixed [001] and [011] orientation when grown on glass substrates at RT. Occasionally $t_{\mathrm{Si}}=14 \AA$ films with a pure (011) orientation are obtained at RT. The variation in texture may be due to changes in film stress under slightly different deposition conditions. Stress induced during deposition has

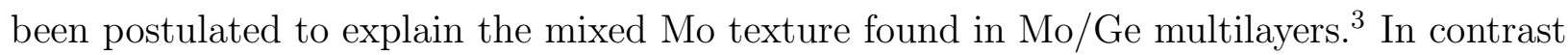


to the thin-Si case, the thicker-Si Fe/Si multilayers which do not show interlayer coupling always have a pure (011) texture. Since the (011) plane is close-packed for the bcc crystal structure, one would expect the (011) orientation to be energetically favored for the Fe in a multilayer with amorphous Si. Films grown at nominal RT on glass or oxidized Si substrates typically had rocking curves about $10^{\circ}$ wide indicating a moderate amount of orientation.

Transmission electron microscopy (TEM) has been used to further investigate the morphology of the films. TEM cross-sectional images of an (Fe30 $\AA / \mathrm{Si} 20 \AA) \times 50$ multilayer and an

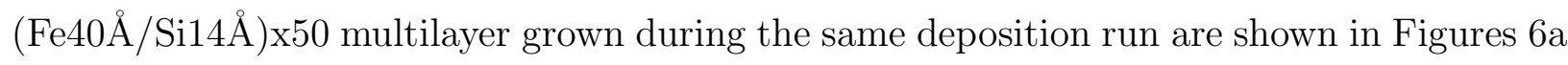
and $6 \mathrm{~b}$, respectively. The most salient features of the (30/20) multilayer are the long lateral continuity of the layers and the smoothness of the interfaces. Since there is no interlayer coherence in the (30/20) film, the crystalline grains have a high aspect ratio. The (40/14) multilayer also has long, continuous layer planes but has rougher interfaces, consistent with the SAXS data.

Transmission electron selected-area diffraction patterns for the (30/20) and (40/14) films are shown in parts c and d of Figure 6. The (30/20) films shows only a $\mathrm{Fe}(011)$ ring, consistent with the high-angle x-ray diffraction scans. The (40/14) film, on the other hand, displays spots corresponding to the (011) and (002) reflections seen using x-rays. The presence of spots rather than rings in the (40/14) image implies the presence of large, oriented crystallites in the film. Most interestingly, the (40/14) image includes a faint spot near what would be the $\mathrm{Fe}(001)$ position were the $\mathrm{Fe}(001)$ peak not forbidden by symmetry in the bcc crystal structure. The (001) peak is allowed in the $\mathrm{B} 2$ and $\mathrm{DO}_{3}$ crystal structures. The B2 structure is found in the equilibrium phase diagram only at $10-22 \%$ Si range of composition, 2 but workers at ETH have grown this crystal structure throughout the range of composition on $\mathrm{Si}$ substrates using $\mathrm{MBE} .33$ The $\mathrm{DO}_{3}$ phase found in the equilibrium phase diagram is $\mathrm{Fe}_{3} \mathrm{Si}$, which is ferromagnetic 32 Clearly a ferromagnetic spacer phase is not consistent with the observation of antiferromagnetic interlayer coupling, although a nonstoichiometric $\mathrm{DO}_{3}$-structure phase might have different magnetic order. The $\mathrm{B} 2$ and $\epsilon$ iron silicide phases have both been previously suggested as possible candidates for the spacer 
layer in AF-coupled Fe/Si multilayers.䏹圂 The position of the (001) TEM spots is not consistent with the $d$-spacings of the $\epsilon$ phase.

According to the powder-diffraction files for the $\mathrm{B} 2$ and $\mathrm{DO}_{3}$ structures, only the (111) peak of the fcc-family $\mathrm{DO}_{3}$ does not coincide with a $\mathrm{B} 2$ peak. The (111) peak would be expected to be very weak in the diffraction patterns formed from cross-sectional specimens of the film. The reason is that a small number of grains contributes to the cross-sectional image, and the probability of sampling a grain with its (111) planes in the observable direction is small because of the random in-plane orientation. Future work will include electron diffraction studies of a (40/14) specimen prepared in the plan-view geometry, where the number of grains which are sampled is considerably larger and the odds of observing the fcc (111) peak are improved.

High-resolution TEM images of the (30/20) and (40/14) multilayers are displayed in Figure 7 . The (30/20) film is shown in 7 a to have a crystalline Fe layer and amorphous spacer layer, similar to the morphology seen before in $\mathrm{Mo} / \mathrm{Si} \mathrm{i}^{3}$. 4 and $\mathrm{Co} / \mathrm{Si}$ multilayers. 1 The (40/14) multilayer in Fig. $7 \mathrm{~b}$ on the other hand is made up entirely of crystalline layers. The coherence between the Fe and silicide spacer is clearly evidenced by the continuity of atomic layer planes from the Fe layer into the spacer. Some crystallites in the (40/14) film extend all the way from the substrate to the surface of the film. The small coherence lengths observed in x-ray diffraction data for the uncoupled thicker-Si films are explained by the presence of the amorphous layers. The lack of crystallinity in the spacer layer of $t_{\mathrm{Si}}=20 \AA$ films is presumably due to insufficient time for full interdiffusion and ordering in the thicker layers. A kinetic mechanism for the lack of crystallization is supported by experiments which show that intentional placement of Fe in the Si layer allows thicker spacer layers to crystallize.25, 35

Another striking feature of the image in Figure $7 \mathrm{~b}$ is the periodic modulation that occurs in the silicide spacer layer. The modulation originates from scattering by inequivalent planes of atoms. Simulation of this image using a multiple-scattering computer calculation may be helpful in positively identifying the crystal structure of the spacer layer phase. 
Dark-field images of the (40/14) multilayer can help answer questions about the texture of the film as well. Figure 8a shows the same bright-field image as in Figure Gb. Dark-field images were formed using (001), (002) and (011) spots from the diffraction pattern shown in Figure 6d. The resulting micrographs are shown in Figures $8 \mathrm{~b}$, c, and d respectively. Panels $\mathrm{a}$ and $\mathrm{b}$ of this figure show the same region of the (40/14) multilayer. The brightness of the spacer layers in this dark-field image demonstrates that the (001) reflection does indeed come from the spacer layer and is not the forbidden (001) spot of bcc Fe. Figures Ec and d also show the same region (although a different region than panels a and b). The bright areas in these two images are the complement of one another; where one is bright, the other is dark and vice versa. The dark-field images in panels $\mathrm{c}$ and $\mathrm{d}$ of Figure 8 demonstrate convincingly that the orientation of the film evolves from predominantly (011) to predominantly (002) as the thickness increases. The reason for the change in orientation with film thickness is not obvious; it may be related to the bilayer-period-number dependence discussed in Section 匹IIC.

The effect of varying the Fe thickness has also been studied. Magnetic properties for films with $20 \AA \leq \mathrm{t}_{\mathrm{Fe}} \leq 50 \AA$ are found to change only slightly in keeping with the expected inverse proportionality of the saturation field with $\mathrm{t}_{\mathrm{Fe}}$. $\mathrm{\text {O }}$ SAXS peaks tend to broaden and even split with increasing Fe thickness, indicating increased disorder in the layering. The splitting of these peaks may indicate different bilayer periods in areas of the film with the (011) and (001) textures. When the Fe is made less than $20 \AA$ thick, the Fe high-angle diffraction peaks disappear and so does the AF coupling. The disappearance of crystalline Fe peaks near $\mathrm{t}_{\mathrm{Fe}}=20 \AA$ is consistent with previous results on evaporated $\mathrm{Fe} / \mathrm{Si}$ multilayers.2. Thus poor crystallinity of the Fe layers appears to suppress the interlayer coupling even when the Si thickness is favorable. The lack of AF coupling in films with poorly crystalline Fe may be related to the lack of a template for the crystalline iron silicide spacer to grow on. 


\section{B. Dependence of Properties on Growth Temperature and Post-Growth Annealing}

Depositing the multilayers at different substrate temperatures is an obvious way of influencing the composition and crystallinity of the spacer layer phase in the Fe/Si multilayers. Fullerton has suggested that the interlayer of Fe/Si multilayers is improved by hightemperature growth. 36 We have grown films on glass substrates at various temperatures between -150 and $+200^{\circ} \mathrm{C}$. The effect of substrate temperature on the interlayer coupling of

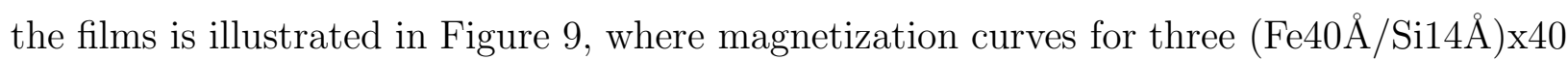
multilayers grown at $-150^{\circ} \mathrm{C},+60^{\circ} \mathrm{C}$ (nominal RT) and $+200^{\circ} \mathrm{C}$ are shown. The data show that as the substrate temperature increases the saturation field increases indicating larger AF coupling. The saturation magnetization also decreases, suggesting a larger degree of interdiffusion in the films grown at higher temperatures.

The suspicion that more interdiffusion occurs at higher substrate temperatures is confirmed by examination of the SAXS spectra for the three films, shown in Figure 10. The film grown at reduced temperature has 7 peaks while the film grown at nominal RT has 5 and the film grown at $+200^{\circ} \mathrm{C}$ has only 4 . Quantitative modelling of low-angle x-ray data has shown that the suppression of higher-order peaks may be due to either interdiffusion or cumulative roughness.28,37 Certainly larger cumulative roughness could also occur at higher growth temperatures, but one would expect very rough growth to suppress AF coupling due to an increased number of pinholes and larger magnetostatic interlayer coupling 39 Since higher growth temperatures seem to enhance rather than suppress the coupling, it seems more likely that high substrate temperatures are promoting interdiffusion rather than roughness. Studies of Mo/Si multilayers showed that a growth temperature of $150^{\circ} \mathrm{C}$ gives maximum SAXS reflectivity, which the authors attribute to greater interface smoothness than for RT deposition. 39 Smaller bilayer periods in multilayers grown at higher temperatures support the claim of increased interdiffusion. Fitting Eqn. 11 to peak positions from Figure 10 gives $\Lambda=52.7,49.3$, and $43.8 \AA$ respectively for the $-150^{\circ},+60^{\circ}$, and $+200^{\circ}$ multilayers versus the nominal value of $54 \AA$. 
Higher substrate temperatures may also promote ordering of the Fe and Si atoms in the crystalline spacer layer. In the fully ordered B2 phase, the Fe and Si atoms sit on different simple cubic sublattices. The sublattice order can occur irrespective of whether or not the Fe to $\mathrm{Si}$ ratio is $1: 1$. It is interesting to speculate whether the AF coupling is dependent on the degree of ordering in the spacer layer. An ordering-dependent coupling seems plausible in light of the Fermi-surface theories of coupling in metal/metal multilayers.40.41 A well-ordered $\mathrm{B} 2$ or $\mathrm{DO}_{3}$ phase would have more well-defined Fermi surface features than a random solid solution. Unfortunately the (001) silicide peak has only been observed by TEM, making experimental attempts to address this issue difficult. Further studies with x-ray diffraction and soft x-ray fluorescence are underway.

The crystallinity of the films also varies with growth temperature. Surprisingly, films grown at both low and high temperatures on glass substrates always have only the (011) texture, while films grown at nominal RT often have mixed (001) and (011) textures. The multilayers deposited on heated and cooled substrates do differ greatly in that those grown at low temperature have amorphous spacer layers, while those grown at high temperatures have long crystalline coherence lengths. The reasons for the strange temperature dependence of growth texture are not understood, although one presumes that they have to do with the kinetics of growth. It is not clear why the (001) texture should appear at all, although it has also been seen in Mo/Ge multilayers 3 An oscillatory dependence of film texture on spacer layer thickness and deposition conditions has been reported for $\mathrm{NiFe} / \mathrm{Cu}$ multilayers grown by IBS. 12 The (001) texture has not been reported in polycrystalline magnetron-sputtered Fe/Si multilayers, and may be due to some peculiarity of IBS growth.

A logical extension to the growth temperature studies is to try annealing the $\mathrm{Fe} / \mathrm{Si}$ multilayers grown at lower substrate temperatures to see if their properties evolve towards those of the multilayers grown at higher temperatures. As far as the magnetic properties

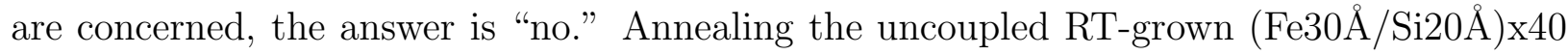

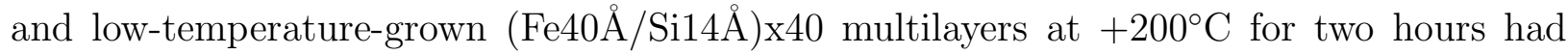
almost no effect on their magnetic properties beyond a slight magnetic moment reduction. 
A subsequent $300^{\circ} \mathrm{C}$ anneal for two hours once more produced a moment reduction and a decrease in coercive field in the uncoupled multilayers. A very low coercive field for annealed $\mathrm{Fe} / \mathrm{Si}$ films is not surprising given the well-known softness of $\mathrm{Fe}-\mathrm{Si}$ alloys. A $300^{\circ} \mathrm{C}$ anneal even eliminated the interlayer exchange coupling of a RT-grown (Fe $40 \AA / \operatorname{Si} 14 \AA) \times 50$ film used as a control. For this $(40 / 14)$ multilayer, the $300^{\circ} \mathrm{C}$ anneal caused the SAXS peaks to narrow and reduced their number from 5 to 4 . At the same time the bilayer period decreased from $49.4 \AA$ to $46.0 \AA$. High-angle x-rays spectra (not shown) indicated that the Fe lattice constant slightly decreased, which is consistent with increased diffusion of Si in the Fe layer.31 These x-ray and magnetization results imply that annealing primarily promotes interdiffusion of the Fe and silicide layers. With sufficient interdiffusion the spacer layer may become ferromagnetic, which would explain the suppression of antiferromagnetic interlayer coupling. These $\mathrm{Fe} / \mathrm{Si}$ multilayers show less thermal stability than $\mathrm{Mo} / \mathrm{Si}$ multilayers with comparable layer thicknesses, which do not show changes in SAXS spectra until $400^{\circ} \mathrm{C} 39$ There was no sign of the solid-state amorphization previously observed in $\mathrm{Fe} / \mathrm{Si}$ multilayers with thicker layers 43

Whatever process occurs during annealing, it does not enhance the interlayer coupling the way that $+200^{\circ} \mathrm{C}$ growth does. This is hardly surprising given that annealing will tend to drive the multilayer towards its equilibrium state, presumably a mixture of different iron

silicide phases. There is no reason to think that the crystalline $\mathrm{Fe} / \mathrm{Fe}_{x} \mathrm{Si}_{1-x}$ multilayer should be an intermediate phase during the annealing. In the future the kinetics of $\mathrm{Fe} / \mathrm{Si}$ multilayer growth at different substrate temperatures will be investigated further by employing an ionassist gun to improve atomic surface mobility.

\section{Dependence of Properties on Number of Bilayers}

One puzzling aspect of the interlayer exchange coupling in the $\mathrm{Fe} / \mathrm{Si}$ system has been the dependence of its strength on the number of bilayers in the multilayer. This trend is illustrated in Figure [1], where magnetization curves for $(\mathrm{Fe} 40 \AA / \mathrm{Si} 14 \AA) \times \mathrm{N}$ multilayers 
with 2, 12 and 25 repeats are displayed. (The 2 -repeat multilayer is just an Fe/Si/Fe trilayer.) Although the trilayer has magnetic properties like bulk Fe, the 25-repeat multilayer data has a magnetization curve similar to the 40-repeat multilayer data shown above. The magnetization curve for the 12-repeat multilayer falls in between that for the thicker and thinner films. Evidence for AF coupling which is stronger near the top of an Fe/Si multilayer than near the substrate has previously been described by Fullerton et al 4 Presumably the increase of coupling with bilayer-number is a manifestation of the same phenomenon. The interlayer coupling in $\mathrm{Co} / \mathrm{Cu}$ multilayers also increases with the number of bilayer periods up to about 25 bilayers. 4

One would not expect interlayer coupling that is quantum-mechanical in nature to be affected much by total film thickness. The unusual thickness dependence therefore raises the question of whether there is quantum-mechanical coupling at all, or whether some other mechanism might determine the shape of the magnetization curves. Disordered magnetic materials such as small amorphous Fe particles can have low remanence and high saturation fields without any layering at all. The magnetization curves of these Fe particles are in fact quite similar to those of the $\mathrm{Fe} / \mathrm{Si}$ multilayers. 46 This resemblance might lead to speculation that the topmost $\mathrm{Fe}$ layers in Fe/Si multilayers are discontinuous and that the magnetic properties are dominated by particle shape. However, the existence of half-order peaks in polarized neutron reflectometry measurements in the IBS-grown Fe/Si multilayers26 and the magnetron-sputtered multilayers 44 gives unambiguous evidence that the magnetic properties are due to magnetic order rather than structural disorder. In addition, TEM pictures such as Figure 6 show that the Fe layers are continuous in films with both high and low saturation fields.

How then does the number of bilayer periods influence the AF coupling strength? It has been suggested that the difference between thin and thick multilayers grown at nominal RT is that the substrates of thick multilayers have time to rise to a higher temperature (about $+60^{\circ} \mathrm{C}$ for our system) during the longer growth. 36 This idea seems reasonable in light of the larger coupling in samples grown on heated substrates as described above. 
In order to investigate this idea, a $(\mathrm{Fe} 100 \AA / \mathrm{Si} 14 \AA / \mathrm{Fe} 100 \AA)$ film was grown on glass at $+200^{\circ} \mathrm{C}$. The magnetization curve for this film is shown in Figure 12. Also shown in this figure are data for a $(\mathrm{Fe} 100 \AA / \mathrm{Si} 14 \AA / \mathrm{Fe} 100 \AA)$ trilayer deposited at nominal RT and for a $(\mathrm{Fe} 100 \AA / \mathrm{Si} 14 \AA / \mathrm{Fe} 100 \AA)$ trilayer deposited at $+200^{\circ} \mathrm{C}$, both grown on a $500 \AA$-thick a-Si buffer. The trilayer deposited directly on glass at elevated temperature has only slightly less remanence and higher saturation field than the trilayer grown at RT whose data are shown in Figure 11. This result implies that it is not substrate temperature alone which causes bilayer-number effects. The magnetization curves of the trilayers grown on buffer layers, on the other hand, look much more like typical $\mathrm{t}_{\mathrm{Fe}}=40 \AA 40$-repeat multilayer results. An epitaxial $(\mathrm{Fe} 100 \AA / \mathrm{Si} 14 \AA / \mathrm{Fe} 100 \AA)$ trilayer grown directly on an $\mathrm{MgO}(001)$ at $+200^{\circ} \mathrm{C}$ substrate also has strong AF coupling (data not shown). Undoubtedly the strong AF coupling of the trilayer grown directly on the $\mathrm{MgO}$ is due the superior surface quality of the single-crystal substrate.

The take-away lesson from all of these results is that substrate roughness is probably responsible for the reduced interlayer coupling in $(\mathrm{Fe} 40 \AA / \mathrm{Si} 14 \AA)$ multilayers with a low number of bilayers. Conformal growth may propagate this roughness up from the substrate into the multilayer. Parkin et al. have found that the interlayer coupling in MBE-grown $\mathrm{Co} / \mathrm{Cu}$ multilayers is very sensitive to the substrate and the buffer layer type, perhaps due to pinholes through the $\mathrm{Cu}$ layers. 4 Presumably thin Fe layers grown directly on glass are so wavy that pinhole and magnetostatic coupling dominate the interlayer interactions for the first few bilayer periods. Recent calculations show that magnetostatic effects associated with propagating roughness can give interlayer ferromagnetic coupling of the same order of magnitude as the coupling derived from quantum-well effects. 3 Ongoing polarized neutron reflectivity experiments may give more information on the variation of the coupling with position in the thicker multilayers.2 2 


\section{Growth on Single-Crystal Substrates}

That Fe films can be grown epitaxially on $\mathrm{MgO}$ and $\mathrm{Al}_{2} \mathrm{O}_{3}$ substrates is well-known. One might therefore expect to be able to grow high-quality Fe/Si superlattices on these substrates. Figure 13a) shows high-angle x-ray diffraction spectra for a purely (001)-oriented

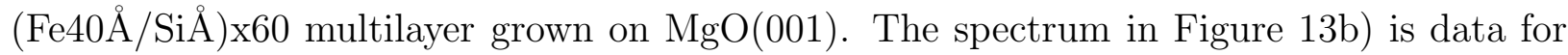

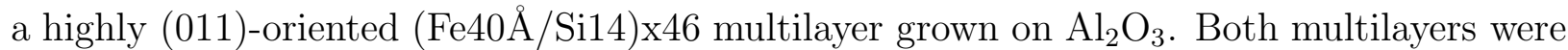
deposited at $+200^{\circ} \mathrm{C}$. Figure 13. $)$ shows a $\phi$ scan for the $\mathrm{MgO}(110)$ and $\mathrm{Fe}(110)$ peaks for the film on the $\mathrm{MgO}$ substrate. These sets of peaks are offset from one another by $45^{\circ}$ in $\phi$, confirming the well-known epitaxial relation $\mathrm{Fe}(001) \| \mathrm{MgO}(001)$ and $\mathrm{Fe}(110) \|$ $\mathrm{MgO}(100) .4$ The $\phi$ scans for the $\mathrm{Al}_{2} \mathrm{O}_{3}$ substrate show that this film is only weakly oriented in-plane. Mattson et al. have previously grown Fe/FeSi multilayers on $\mathrm{Al}_{2} \mathrm{O}_{3}$, but they did not comment on the orientation of the multilayer.49 Rocking curves widths for both films are about $1^{\circ}$ wide, indicating a considerably smaller mosaic than for the multilayers grown on glass. SAXS data for the multilayers on single-crystal substrates are comparable to the data for films grown on glass.

The films grown on $\mathrm{MgO}$ are the only purely (001)-textured Fe/Si multilayers produced by IBS so far. Dekoster et al. have grown epitaxial Fe/FeSi multilayers on $\mathrm{MgO}(001)$ by MBE, but they do not present any x-ray diffraction data or magnetization curves.34 Magnetization curves of films grown on single-crystal substrates (not shown) are qualitatively similar to those grown on glass or oxidized Si substrates. The only differences are that the saturation fields are higher for the epitaxial samples and that magnetocrystalline anisotropy effects are observed. The magnetocrystalline anisotropy energies of epitaxial trilayers grown on $\mathrm{MgO}$ and Ge are similar to bulk Fe.5

The shape of the high-angle peaks plus superlattice satellites are described by a theory due to Fullerton et al.28 Application of this theory to the Fe/Si multilayers is difficult because the silicide lattice constant, the thickness of the remaining pure Fe and the thickness of the silicide spacer can be estimated only roughly. A precise determination of the silicide lattice 
constant should make a quantitative analysis of these satellite features possible.

\section{DISCUSSION}

Fe and Si appear to be the only known transition-metal/semiconductor combination in which the two elements interdiffuse to form a crystalline spacer layer with coherent interfaces. The reasons why this unusual morphology occurs in the Fe/Si system are unknown but likely involve a high rate of Fe diffusion into a-Si and a low heat of crystallization of the iron silicide compound. A detailed discussion of these issues is beyond the scope of this paper.

Three different crystal structures have been proposed for the crystalline spacer layer of the Fe/Si multilayers. The $\epsilon$ phase can be eliminated on the basis of the electron diffraction patterns and TEM dark field images presented here. The $\mathrm{B} 2$ and $\mathrm{DO}_{3}$ crystal structures are better lattice-matched to Fe than $\epsilon$-FeSi or $\alpha$ - and $\beta$-FeSi ${ }_{2}$. The lattice constant of the B2 phase was reported by Mäder and coworkers to be $2.77 \AA$, only $3.1 \%$ different from Fe.51 The lattice constant of the $\epsilon$ phase is $4.46 \AA$, 5 wich matches the $\mathrm{Fe}(110)$ plane only in the energetically unfavorable (210) direction. 35

Recent conversion-electron Mössbauer data are interpreted in support of the B2 crystal structure, although the possibility of the $\mathrm{DO}_{3}$ phase was not considered in that study.34 It is plausible that the $\mathrm{B} 2$ or $\mathrm{DO}_{3}$ structures form in rapid, far-from-equilibrium growth conditions because of their small unit cells. Since silicon deposited at low substrate temperatures is amorphous, the most likely scenario is the following. Silicon deposited on a crystalline Fe layer goes down amorphous and diffuses only slightly into the Fe. Subsequently deposited Fe atoms diffuse rapidly into the amorphous $\mathrm{Si}$, analogous to what happens during the growth of Mo/Si multilayers 13, 14 During the diffusion of Fe into Si, crystallization of the silicide occurs, possibly driven by the heat of mixing or by the kinetic energy of the incident Fe atoms. Growth of the crystalline phase may proceed upward from the lattice-matched Fe template, or downward from the atomically bombarded film surface. If the growth of the crystalline silicide phase proceeds downward from the film surface, one might expect to see 
some crystalline silicide in the high-resolution TEM image for the $\mathrm{t}_{\mathrm{Si}}=20 \AA$ film (Figure $7 \mathrm{~b}$ ). The lack of any evidence for crystalline silicide in this image suggest that the crystallization proceeds upward from the iron/silicide interface, not downward from the film surface.

It is difficult to determine how realistic this model for growth of the crystalline silicide is since the $\mathrm{Fe} / \mathrm{Fe}-\mathrm{Si}$ and $\mathrm{Si}-\mathrm{Fe} / \mathrm{Fe}$ interfaces appear identical in Figure $7 \mathrm{~b}$. In contrast, the Mo/Si and Si/Mo interfaces in Mo/Si multilayers appear quite different from one another.13/14 In the Mo/Si multilayers, an amorphous $\mathrm{MoSi}_{2}$ region appears which is thicker at the Mo/Si interface than at the Si/Mo interface. Detailed TEM studies of multilayers with $\mathrm{t}_{\mathrm{Si}}$ larger than $20 \AA$ may help to answer whether amorphous silicides can occur in IBS-grown $\mathrm{Fe} / \mathrm{Si}$ multilayers.

Using the B2 phase lattice constant reported by the Zürich group, 51 we can estimate the expected bilayer period of a nominal Fe/Si multilayer in which Fe atoms diffuse into the Si layer up to a 1:1 stoichiometry. The spacing between the Fe and $\mathrm{Fe}_{\mathrm{x}} \mathrm{Si}_{1-\mathrm{x}}$ layers is taken as the average of the interplanar spacings of the two materials. The result of this rough calculation is that an $(\mathrm{Fe} 40 \AA / \mathrm{Si} 14 \AA)$ multilayer which interdiffuses up to the $1: 1$ stoichiometry should form a $(\mathrm{Fe} 33.2 \AA / \mathrm{FeSi} 16.3 \AA)$ multilayer with a bilayer period of $49.4 \AA$. The missing bilayer period predicted from this model is $4.6 \AA$, in the middle of values on the $\mathrm{x}$-axis of Figure 4 . One can also calculate the expected magnetic moment reduction assuming that Fe atoms in the silicide layer have no moment and those in the Fe layer have their full moment. Under this assumption a calculation predicts $8.2 \AA$ of missing Fe moment, slightly lower than indicated in Figure 4 . This calculation neglects the possibility that some Fe atoms in the Fe layer with Si near neighbors may have reduced magnetic moments.

In the discussion above the possibility has not been mentioned that the missing bilayer period and magnetic moment are due to an inaccurate thickness calibration. This explanation is contradicted by magnetization and x-ray diffraction measurements on Fe/Ge multilayers, where measured magnetic moments and bilayer periods are in much closer agreement with nominal values than for Fe/Si 50 The improved agreement in the case of Fe/Ge multilayers 
suggests that interdiffusion is less important in multilayers with Ge spacer layers than in multilayers with Si spacers.

The main point is that the formation of the B2 silicide does qualitatively explain the bilayer period reduction observed in the Fe/Si multilayers. The underlying reason for the bilayer period reduction is that the silicide which forms is denser than both Fe and Si. This situation is similar to that observed in other metal/Si multilayers 10,14 except that in the other multilayers the silicide remains amorphous.

Confirmation that the spacer layer phase has the $\mathrm{B} 2$ or $\mathrm{DO}_{3}$ structure is important for understanding the coupling mechanism in these compounds. Both the $\mathrm{B} 2$ and $\mathrm{DO}_{3}$ phases are known to be metallic for some ranges of composition. 15,16 Thus the present results and those of other workers5 34 suggest that $\mathrm{Fe} / \mathrm{Si}$ is really a metal/metal multilayer. The origin of the interlayer coupling is then likely to be described by the same theories as describe coupling in $\mathrm{Co} / \mathrm{Cu}$ and $\mathrm{Fe} / \mathrm{Cr}$ multilayers.40, $11 \mathrm{Fe} / \mathrm{Si}$ multilayers may therefore not be a good test case for theories which model interlayer exchange coupling across insulators.80

In the discussion above the possibility has been neglected that the amorphous spacer layer in the thick-Si films may also be metallic. If both the thick amorphous spacers and the thin crystalline spacers are metallic silicides, then it must be the crystallinity that is the essential feature for the existence of AF interlayer coupling. Up to now there have been no reports of AF coupling across amorphous metallic spacer layers. Toscano et al. have reported AF coupling across amorphous silicon spacer layers. were prepared at low temperature so as to suppress interdiffusion. 1 the character of AF coupling in the a-Si spacer trilayers is likely quite different than in the multilayers described in this study, where substrate heating increases the strength of coupling.

At the moment there is no direct evidence regarding the metallic or insulating nature

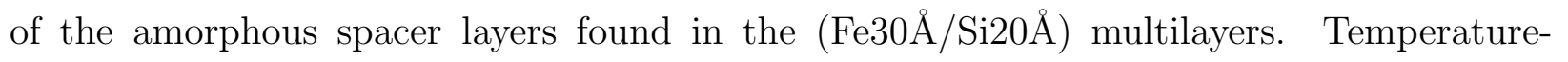
dependent current-in-plane resistivity measurements suggest that both crystalline and amorphous spacer layers in $\mathrm{Fe} / \mathrm{Si}$ multilayers are poorly conducting. $50 \mathrm{Fe}_{70} \mathrm{Si}_{30}$ and $\mathrm{Fe}_{65} \mathrm{Si}_{35}$ amorphous alloys have a temperature-independent resistivity, suggesting non-metallic behavior. 52 


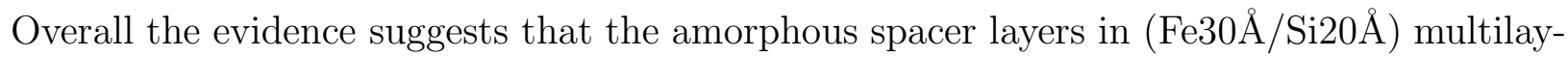
ers are not metallic, but spectroscopic measurements like soft x-ray fluorescence 24 are needed for confirmation. The interesting question as to whether there can be AF interlayer coupling across an amorphous metal spacer layer must then be left for another study.

\section{CONCLUSIONS}

An extensive study of the growth of Fe/Si multilayers by ion-beam sputtering has been performed. The crystalline quality of the films is better when they are grown with thick Fe layers, with thin Si layers, at high temperature, and on single-crystal substrates. Improved growth conditions lead to higher saturation fields and lower remanence in magnetization curves. Measured bilayer periods are consistently shorter in these multilayers than the nominal value, suggesting formation of a dense silicide phase in the spacer layer. Despite considerable interdiffusion in the multilayers, a strong composition modulation along the growth direction is maintained as evidenced by SAXS measurements.

There are two surprising results from this study. One is that the films grow on glass with a mixed (011) and (001) texture near nominal RT and with a pure (011) texture at higher and lower temperatures. The other surprise is that the strength of the interlayer coupling depends strongly on the number of bilayer periods in films with thin Fe layers. This latter result is explained on the basis of substrate surface roughness.

Unraveling the behavior of the Fe/Si multilayer system has proven to be a considerably more complex task than understanding the $\mathrm{Fe} / \mathrm{Cr}$ or $\mathrm{Co} / \mathrm{Cu}$ multilayer systems. The reason is that compound formation at the $\mathrm{Fe} / \mathrm{Si}$ interface is crucial to understanding the AF interlayer coupling. Identification of possibly disordered phases in the spacer layer of a multilayer continues to be an experimental challenge. Mounting evidence suggests that the spacer layer in the AF-coupled Fe/Si multilayers is metallic and crystalline and that the Fe/Si interlayer coupling therefore has the same origin as in metal/metal multilayers. 
Acknowledgements We would like to thank P.E.A. Turchi, T.W. Barbee Jr., T.P. Weihs, E.E. Fullerton, Y. Huai and E.C. Honea for helpful discussions, and B.H. O'Dell and S. Torres for technical assistance. Further thanks go to C.-T. Wang of Stanford for the four-circle x-ray diffractometry and to Sandia National Lab for use of their electron microscope for HREM work. Part of this work was performed under the auspices of the U.S. Department of Energy by LLNL under contract No. W-7405-ENG-48. 


\section{REFERENCES}

* chaiken@llnl.gov

† michel@cmsgee.llnl.gov

‡ mark_wall@internetqm.llnl.gov

${ }^{1}$ S.T. Ruggiero, T.W. Barbee, Jr., and M.R. Beasley, Phys. Rev. B26, 4894 (1982).

${ }^{2}$ T.W. Barbee, S. Mrowka, and M.C. Hettrick, Appl. Opt. 24, 883 (1985).

${ }^{3}$ L.C. Wilson, Ph.D. thesis, Stanford University, 1993.

${ }^{4}$ S. Toscano, B. Briner, H. Hopster, and M. Landolt, J. Magn. Magn. Matls. 114, L6 (1992).

${ }^{5}$ E.E. Fullerton, J.E. Mattson, S.R. Lee, C.H. Sowers, Y.Y. Huang, G. Felcher, S.D. Bader, and F.T. Parker, J. Appl. Phys. 73, 6335 (1993).

${ }^{6}$ S. Demokritov, J.A. Wolf, and P. Grünberg, Europhys. Lett. 15, 881(1991).

${ }^{7}$ D.H. Mosca, F. Petroff, A. Fert, and P.A. Schroeder, J. Magn. Magn. Matls. 94, L1 (1991).

${ }^{8}$ P. Bruno, Phys. Rev. B49, 13231 (1994).

${ }^{9}$ Z.-P. Shi, Rajiv R.P. Singh, and B.M. Klein, Europhys. Lett. 29, 585 (1995).

${ }^{10}$ E.E. Fullerton, J. Pearson, C.H. Sowers, S.D. Bader, X.Z. Wu, and S.K. Sinha, Phys. Rev. B48, 17432 (1993).

${ }^{11}$ H. Miura, E. Ma, and C.V. Thompson, J. Appl. Phys. 70, 4287 (1991).

${ }^{12}$ W.H. Wang, H.Y. Bai, and W.K. Wang, Matls. Sci. Eng. 179A, 229 (1994).

${ }^{13}$ D.G. Stearns, R.S. Rosen, and S.P. Vernon, J. Vac. Sci. Techn. A9, 2662 (1991).

${ }^{14}$ K. Holloway, K.B. Do, and R. Sinclair, J. Appl. Phys. 65, 474 (1989).

${ }^{15}$ H. von Känel, K.A. Mäder, E. Müller, N. Onda, and H. Sirringhaus, Phys. Rev. B45, 13807 (1992). 
${ }^{16}$ J. Kudrnovský, N.E. Christensen, and O.K. Andersen, Phys. Rev. 43, 5924 (1991).

${ }^{17}$ Made by Commonwealth Scientific Corporation, 500 Pendleton St., Alexandria, VA 22314.

${ }^{18}$ MKS Instruments, 6 Shattuck Rd., Andover, MA 01810.

${ }^{19}$ MDC, 23842 Cabot Blvd., Hayward, CA 94545.

${ }^{20}$ A. Chaiken, E.C.Honea, W.S. Rupprecht, S. Torres, and R.P. Michel, Rev. Sci. Instr. 65, 3870 (1994).

${ }^{21}$ Keithley Instruments, 440 Myles Standish Blvd., Taunton, MA 02780.

${ }^{22}$ A.I. Kingon, O. Auciello, M.S. Ameen, S.H. Rou, and A.R. Krauss, Appl. Phys. Lett. 55, 301 (1989).

${ }^{23}$ R.F.C. Farrow, G.R. Harp, R.F. Marks, T.A. Rabedeau, J. Cryst. Growth 133, 47 (1993).

${ }^{24}$ A. Chaiken, R.P. Michel, J.A. Carlisle and M.A. Wall, Proceedings of 1995 Magnetism and Magnetic Materials Conference, submitted.

${ }^{25}$ C.L. Foiles, M.R. Franklin, and R. Loloee, Phys. Rev. B50, 16070 (1994).

${ }^{26}$ R.P. Michel, A. Chaiken, J.W. Dykes, J.F. Ankner and H.J. Kaiser, Proceedings of 1995 Magnetism and Magnetic Materials Conference, submitted.

${ }^{27}$ K. Inomata, K. Yusu, and Y. Saito, Jpn. J. Appl. Phys. 33, L1670 (1994).

${ }^{28}$ E.E. Fullerton, I.K. Schuller, H. Vanderstraeten, and Y. Bruynseraede, Phys. Rev. 45, 9292 (1992).

${ }^{29}$ C. Dufour, A. Bruson, G. Marchal, B. George, and Ph. Mangin, J. Magn. Magn. Matls. 93, 1991.

${ }^{30}$ J.F. Ankner, C.F. Majkrzak, and H. Homma, J. Appl. Phys. 736436 (1993).

${ }^{31}$ C.L. Foiles, M.R. Franklin, and R. Loloee, Mat. Res. Soc. Proc. 382, to be published 
(1995).

${ }^{32}$ Binary Alloy Phase Diagrams, T.B. Massalski, editor, ASM International, Materials Park, OH, 1990.

${ }^{33}$ N. Onda, H. Sirringhaus, E. Muller, and H. von Känel, J. Cryst. Growth 12, 634 (1993).

${ }^{34}$ J. Dekoster, A. Vantomme, S. Degroote, R. Moons, and G. Langouche, Mat. Res. Soc. Proc. 382, to be published (1995).

35 J.E. Mattson, S. Kumar, E.E. Fullerton, S.R. Lee, C.H. Sowers, M. Grimsditch, S.D. Bader and F.T. Parker, Phys. Rev. Lett. 71, 185 (1993).

${ }^{36}$ E.E. Fullerton, private communication.

${ }^{37}$ A.P. Payne and B.M. Clemens, Phys. Rev. 47, 2289 (1993).

${ }^{38}$ D. Altbir, M. Kiwi, R. Ramírez and I.K. Schuller, preprint.

${ }^{39}$ H.-J. Stock, U. Kleineberg, B. Heidemann, K. Hilgers, A. Kloidt, B. Schmiedeskamp, U. Heinzmann, M. Krumrey, P. Müller and F. Scholze, Appl. Phys. A58, 371 (1994).

${ }^{40}$ P. Bruno and C. Chappert, Phys. Rev. B46 261 (1992).

${ }^{41}$ M.D. Stiles, Phys. Rev. B48, 7238 (1993).

${ }^{42}$ R. Nakatani, T. Dei, and Y. Sugita, J. Appl. Phys. 73, 6375 (1993).

${ }^{43}$ A. Gupta, G. Principi, R. Gupta, A. Maddalena, F. Caccavale, and C. Tosello, Phys. Rev. B50, 2833 (1994).

${ }^{44}$ E.E. Fullerton, J.E. Mattson, S.R. Lee, and C.H. Sowers, J. Magn. Magn. Matls. 117, L301 (1992).

${ }^{45}$ H.A.M. van den Berg and G. Rupp, IEEE Trans. Magn. 30, 809 (1994).

${ }^{46}$ M.W. Grinstaff, M.B. Salamon, and K.S. Suslick, Phys. Rev. B48, 269 (1993). 
47 S.S.P. Parkin, R.F. Marks, R.F.C. Farrow, and G.R. Harp, Phys. Rev. B46, 9262 (1992).

${ }^{48}$ N. Metoki, M. Hofelich, T. Zeidler, and T. Muhge, J. Magn. Magn. Matls. 121, 137 (1993).

${ }^{49}$ J.E. Mattson, E.E. Fullerton, S. Kumar, S.R. Lee, C.H. Sowers, M. Grimsditch, S.D. Bader and F.T. Parker, J. Appl. Phys. 75, 6169 (1994).

${ }^{50}$ R.P. Michel and A. Chaiken, unpublished.

${ }^{51}$ K.A. Mäder, H. von Känel, and A. Baldereschi, Phys. Rev. B48, 4364 (1993).

${ }^{52}$ G. Marchal, Ph. Mangin, and Chr. Janot, Sol. State. Comm. 18, 739 (1976). 


\section{FIGURES}

FIG. 1. Schematic plan of the ion-beam sputtering system.

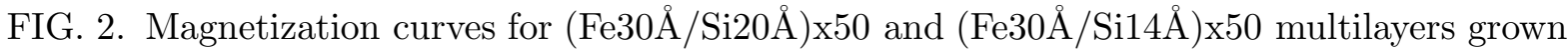
on glass substrates at nominal RT during the same deposition run. Plotted on the y-axis is the observed magnetization of the films divided through by the calculated magnetization of an

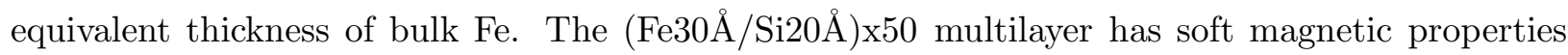

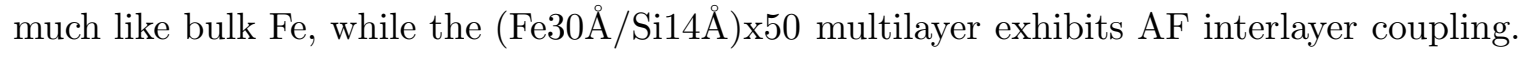

FIG. 3. X-ray diffraction spectra at small-angle for the same films whose magnetization curves are shown above. Broader peaks show that there is more disorder in layering for the AF-coupled film with $\mathrm{t}_{\mathrm{Si}}=14 \AA$. Using Equation 1, these data give bilayer periods $\Lambda=41.82 \AA$ for the nominal

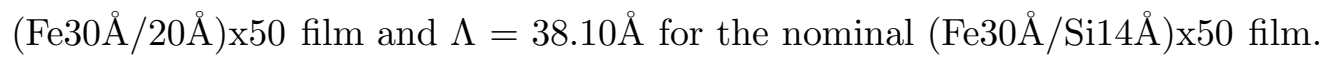

FIG. 4. Missing Fe magnetic moment expressed as an equivalent thickness of Fe plotted versus missing bilayer period as obtained from fits to small-angle x-ray diffraction data. Symbols indicate different nominal Si layer thicknesses and different film textures. The film labelled "LN" was grown on a LN-cooled substrate; all others were grown at nominal RT. All multilayers have 40 or 50 repeats and were grown on either glass or oxidized Si substrates.

FIG. 5. High-angle spectra for two Fe/Si multilayers showing the Fe (011) and (002) peaks. The $\mathrm{t}_{\mathrm{Si}}=20 \AA$ film is predominantly (011)-textured, while the AF-coupled film with $\mathrm{t}_{\mathrm{Si}}=14 \AA$ has mixed (011) and (001) textures. No x-ray diffraction peaks which could be indexed to crystalline

silicon or silicide spacer layer phases have been observed in any Fe/Si multilayer. A superlattice satellite just below the $\mathrm{Fe}(002)$ peak is labelled "-1." 
FIG. 6. Cross-sectional TEM images ( $\mathrm{a}$ and b) and selected area diffraction patterns (c and d) for the same $(\mathrm{Fe} 30 \AA / \mathrm{Si} 20 \AA) \times 50$ multilayer and a $(\mathrm{Fe} 40 \AA / \mathrm{Si} 14 \AA) \times 50$ multilayer grown that shows strong AF coupling. a) and b) show that the Fe/Si multilayers have layers which are continuous for large lateral distances. There is no sign of propagating roughness or columnar growth. c) The (30/20) multilayer shows only an Fe(011) ring. d) The (40/14) film shows (011) and (002) spots plus a faint spot at the (001) position (indicated by an arrow).

FIG. 7. High-resolution TEM images of the same films whose low-resolution images are shown

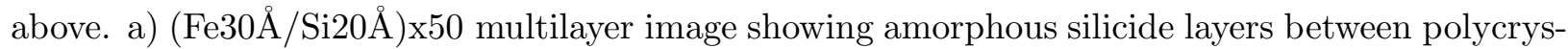

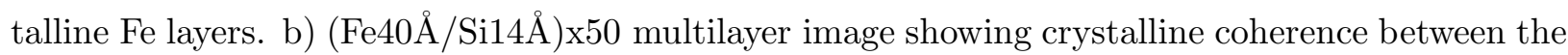
polycrystalline Fe layers and iron silicide spacer layers. There is no amorphous layer present.

FIG. 8. a) The same bright field TEM micrograph of the (Fe $40 \AA / \mathrm{Si} 14 \AA) \times 50$ multilayer as is shown in Figure 6b. b) A dark-field image of the same region of the (40/14) multilayer. This dark-field image was formed using the (001) reflection. Comparison with the bright field image shows that the (001) reflection originates from the Si substrate and the spacer layers. c) and d) Dark-field images formed from (002) and (011) reflections. Image c) shows that planes with (002) orientation predominate near the film surface. Image d) shows that planes with (011) orientation predominate near the substrate. The film surface is on the top of all these images.

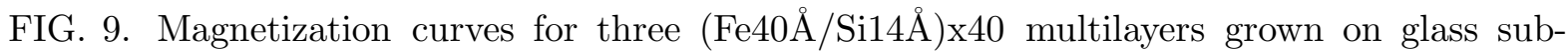
strates at $-150^{\circ},+60^{\circ} \mathrm{C}$ and $+200^{\circ} \mathrm{C}$. The increase of the saturation field with increasing substrate temperature indicates an increase in AF coupling. Note that the saturation magnetization also decreases slightly with increasing substrate temperature.

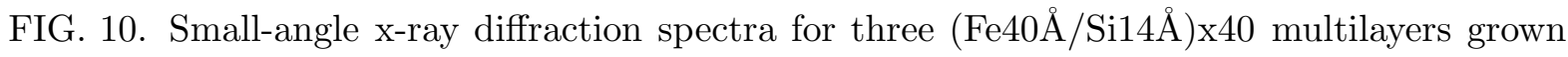
on glass substrates at $-150^{\circ} \mathrm{C},+60^{\circ} \mathrm{C}$ and $+200^{\circ} \mathrm{C}$. The disappearance of higher-order peaks at higher substrate temperatures is an indication of greater interdiffusion. 


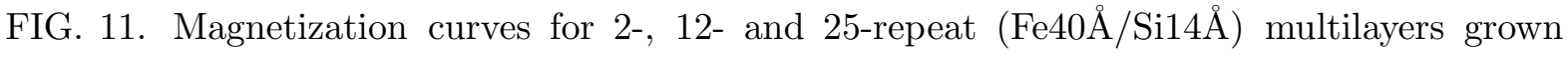
during the same deposition run at nominal RT on glass substrates. The 2-repeat multilayer (really an $\mathrm{Fe} / \mathrm{Si} / \mathrm{Fe}$ trilayer) shows no signs of AF coupling. The 12-repeat multilayer appears to have a smaller coupling than the 25-repeat one.

FIG. 12. Magnetization curves of three (Fe/Si/Fe) trilayers. The open circles are data for an

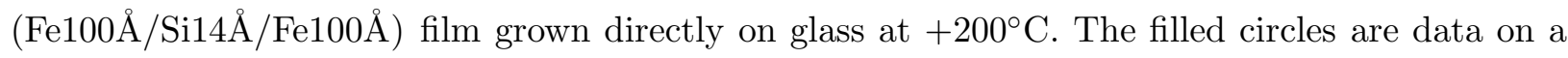

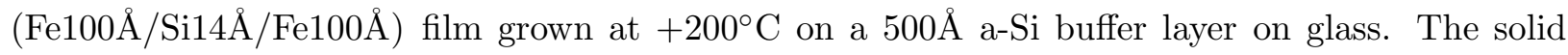
curve is for a $(\mathrm{Fe} 100 \AA / \mathrm{Si} 14 \AA / \mathrm{Fe} 100 \AA)$ film grown at nominal RT on a $500 \AA$ a-Si buffer layer on glass. The coupling is stronger in the film grown at high temperature on a buffer than in either of the other two films.

FIG. 13. High-angle x-ray diffraction spectra from Fe/Si multilayers grown on single-crystal substrates. a) Data for a $(\mathrm{Fe} 40 \AA / \mathrm{Si} 14 \AA) \times 60$ multilayer grown on $\mathrm{MgO}(001)$. The $\mathrm{Fe}(002)$ peak is shown with 5 satellites centered at $64.77^{\circ}$. b) Data for a $(\mathrm{Fe} 40 \AA / \mathrm{Si} 14 \AA) \times 46$ multilayer grown on $\mathrm{Al}_{2} \mathrm{O}_{3}(0 \overline{2} 11)$. Visible in the spectrum are the $\mathrm{Al}_{2} \mathrm{O}_{3}(0 \overline{2} 11)$ peak at $37.79^{\circ}$ and the $\mathrm{Fe}(011)$ peak centered at $44.99^{\circ}$ with its 4 satellites. c) $\phi$ scans plotted on a logarithmic scale for the $\mathrm{MgO}$ and Fe

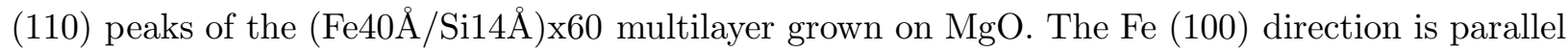
to the $\mathrm{MgO}(110)$, as expected, but a small amount of material with a secondary orientation is also visible. 